\title{
Corona im Knast
}

\author{
Auswirkungen der COVID-19-Pandemie auf die Soziale Arbeit im Justizvollzug \\ des Landes Nordrhein-Westfalen
}

Seit die ersten Corona-Infektionen in Deutschland auftraten, mussten sich die Justizvollzugseinrichtungen auf eine Situation vorbereiten, die trotz vorhandener Pandemiepläne nicht durchzuplanen ist - Dinge, die gestern noch galten, hatten sich am nächsten Tag bereits geändert. Die Lage entwickelt sich extrem dynamisch. Insoweit ist es im Justizvollzug nicht anders als "draußen". Dennoch ist die Bewältigung der Corona-Krise im Justizvollzug eine besondere und extreme Herausforderung.

$\mathrm{D}$ ie spezifische Herausforderung liegt darin, in einer totalen Institution, in der - zum Teil gefährliche - Menschen nicht freiwillig untergebracht sind, unter stark einschränkenden Bedingungen, die Balance zwischen den Betreuungsbedürfnissen der Inhaftierten, der langfristigen Sicherung des Personaleinsatzes, den bestehenden Sicherheitserfordernissen und Anforderungen des Infektionsschutzes herzustellen. Die für die Inhaftierten einschränkenden Maßnahmen haben - je länger sie andauern - unmittelbaren Einfluss auf das Klima in den Justizvollzugsanstalten. Die Sorge, dass eine brisante Entwicklung eintreten könnte, ist bei allen Beteiligten trotz guter Vorbereitung vorhanden.

Als dieser Beitrag geschrieben wurde, waren zwei Inhaftierte im nordrhein-westfälischen Justizvollzug bekanntermaßen an COVID-19 erkrankt. Zum Glück im offenen Vollzug, in dem die Belegungszahlen niedriger und die Handlungsoptionen größer sind. Die Infektionsgefahr kommt von außen: von Bediensteten, von externen Mitarbeiter_innen, von Lieferant_innen, Be-

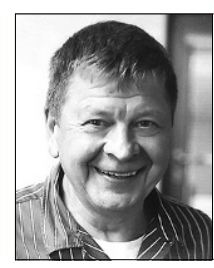

\section{Stephan Schlebusch}

Duisburg, Deutschland

*1959, Diplom-Sozialarbeiter, seit 1984 im Strafvollzug des Landes Nordrhein-Westfalen, seit 2019 Leiter des Fachbereichs Sozialdienst im Justizvollzug des Landes Nordrhein-Westfalen. stephan.schlebusch@jva-duisburg-hamborn.nrw.de

Zusammenfassung Die Corona-Pandemie hat gravierende Auswirkungen auf das System Justizvollzug, die Soziale Arbeit und das Übergangsmanagement. Kriseninterventionen statt Behandlungsangebote sind angesagt. Die Pandemie zeigt die Schwachstellen im gesellschaftlichen System radikal auf. Betroffen sind sowohl Klient_innen als auch Mitarbeiter_innen.

Schlüsselwörter Soziale Arbeit im Justizvollzug, Folgen der Corona-Pandemie auf den Justizvollzug, Übergangsmanagement, Integration von Inhaftierten sucher_innen und Neuinhaftierten, wobei die Zahl der Untersuchungsgefangenen kaum steuerbar ist.

Das Ministerium der Justiz des Landes NordrheinWestfalen ist bemüht, durch zahlreiche Erlasse ${ }^{1}$ Rahmenvorgaben zu setzen. Bei Ermessensspielräumen obliegt die Umsetzung der Erlasse aufgrund ihrer Organisationshoheit den Leitungen der Justizvollzugsanstalten (JVA). Deshalb besteht unter der sich permanent verändernden Situation ein sehr heterogenes, von JVA zu JVA unterschiedliches Bild. Dieses kann beispielsweise bestimmt sein von den unterschiedlichen Vollzugsformen (z. B. Jugend- oder Erwachsenenvollzug, offener oder geschlossener Vollzug ...), von regionalen Aspekten, der Personalsituation oder dem institutionellen Krisenmanagement.

Um Quarantänestationen und Isolationsbereiche für infizierte Gefangene und Beobachtungsabteilungen für Neuinhaftierte einzurichten, war es erforderlich, die Zahl der Inhaftierten und der Neuaufnahmen zu reduzieren. Durch einen Vollstreckungsaufschub ${ }^{2}$ im Jugendarrest, bei Ersatzfreiheitsstrafen und kürzeren Freiheitsstrafen sowie durch eine Haftunterbrechung ${ }^{3}$ nach $\$ 455$ a I StPO bei Inhaftierten, die eine Ersatzfreiheitsstrafe oder eine Freiheitsstrafe bis maximal 18 Monate verbüßen und bis zum 31.07.2020 entlassen worden wären, gelang es, die Zahl der in NRW Inhaftierten von 15.338 am 29.02.2020 auf 14.255 am 31.03.2020 zu senken. Trotzdem weisen die meisten geschlossenen Anstalten noch Belegungsquoten um die $90 \%$ auf.

Der Besuch von Angehörigen wurde für die Inhaftierten weitgehend eingestellt, vollzugsöffnende Maßnahmen wurden ausgesetzt, Verlegungen zwischen den Anstalten gestoppt und externe Stellen erhalten in den Anstalten nur im Ausnahmefall Einlass ${ }^{4}$. Einschneidende Maßnahmen der Kontaktreduzierung, die das Infektionsrisiko verringern sollen, die aber auch eine zusätzliche soziale Dynamik in den Anstalten entstehen lassen kann. 
Der Justizvollzug gehört zu den systemrelevanten Bereichen, in dem Personal für sichernde, versorgende und betreuende Aufgaben zur Verfügung stehen muss. Es gilt, bei sich möglicherweise verschärfenden Bedingungen, hinreichend Personal zur Aufrechterhaltung des Dienstbetriebs zur Verfügung zu haben. Vor dem Hintergrund, dass sich Bedienstete in Quarantäne befinden oder aufgrund von Vorerkrankungen freigestellt sind, haben einige Anstalten weitsichtig ein Schichtsystem eingeführt, bei dem Bedienstetengruppen im Wechsel eine Woche vor Ort arbeiten und die Folgewoche Heimarbeit verrichten. Durch die Trennung der Gruppen ist im Infektions-/Quarantänefall ein Personalersatz gesichert.

\section{Behandlungsangebote sind in Corona-Zeiten sekundär}

Was hat all dies mit Sozialer Arbeit im Justizvollzug zu tun? Soziale Arbeit im Justizvollzug ist ihrem Selbstverständnis nach Teil des Justizvollzugs ${ }^{5}$. In ihrem systemischen Ansatz zielt sie darauf ab, den Vollzug weiterzuentwickeln, strafende Aspekte in den Hintergrund zu drängen, die Institution zu humanisieren und fördernde Angebote auszugestalten. Als Teil des Systems unterliegt sie den institutionellen Rahmenbedingungen und ist wie alle anderen Berufsgruppen auch - zur interdisziplinären Zusammenarbeit verpflichtet.

Dies gilt zurzeit in besonderem Maße: im Knast sitzen alle in einem Boot. Vor dem Hintergrund der Corona-Krise bedeutet dies, dass vom Sozialdienst in dieser Zeit auch betreuerische Aufgaben vorübergehend übernommen werden, die nicht zu dem in den Richtlinien abgebildeten, umfassenden Aufgabenprofil ${ }^{6}$ gehören. Die Richtlinien beschreiben als „Kernaufgaben des Sozialdienstes (die) fachliche Diagnostik und Beratung, Behandlung, Vernetzung und Vermittlung sozialer Hilfen" "7.

Bei den Gefangenen besteht ein ausgeprägtes Informations- und Gesprächsbedürfnis zu der Viruserkrankung und große Sorge um das Wohlergehen von Angehörigen. So informiert der Soziale Dienst über das Krankheitsbild, über Anzeichen und Verhaltensregeln, führt viele entlastende Gespräche und ermöglicht die telefonische Kontaktaufnahme zu Angehörigen. Bei Verständigungsproblemen werden in zahlreiche Sprachen übersetzte Merkblätter ausgehändigt. Im Rahmen der Freizeitgestaltung werden Angebote entwickelt. Der Sozialdienst vermittelt Wissen über die Erkrankung und erklärt damit zusammenhängende Entscheidungen. Durch den transparenten Umgang sorgt er für soziale Befriedung. $\mathrm{Zu}$ Corona-Zeiten ist der Sozialdienst das Schmiermittel im Getriebe.
Die Ausgestaltung der eigentlich dem Sozialdienst obliegenden Aufgaben ${ }^{8}$ ist in den Anstalten sehr unterschiedlich. Um den Gefangenen möglichst viel Normalität zu vermitteln und keine Unruhe aufkommen zu lassen, wird in einigen Anstalten vom Sozialdienst erwartet, das „normale Programm“ zu fahren: Vollzugpläne aufstellen, Berichte schreiben, Einzelgespräche führen, Gruppenangebote durchführen, Entlassungen vorbereiten ...

In anderen Anstalten wird mehr Wert auf die Akutbetreuung gelegt und Gruppenangebote werden in Anbetracht der häufig beengten Situation durch enge Flure und kleine Gruppenräume heruntergefahren. Rollenspiele im Sozialen Training oder Anti-Aggressivitätstraining ohne körperliche Nähe durchzuführen, ist nicht möglich. Social Distancing ist in Einrichtungen, in denen man auf engen Raum zusammenlebt, schwierig. Letztlich haben einige Anstalten sinnvollerweise - u. a. den Sozialdienst - in Gruppen aufgeteilt, die im wöchentlichen Wechsel arbeiten bzw. im Homeoffice sind. Hier geht es darum, die Zahl der physischen Kontakte zu reduzieren, mögliche Infektionswege zu unterbrechen und bei pandemiebedingten Ausfällen eine Personalreserve zu besitzen.

Die Angebote externer Mitarbeiter_innen der freien Wohlfahrtspflege, z. B. in der Sucht- oder Schuldnerberatung, finden in den Anstalten nicht mehr statt. Aus Sorge um das Wohl ihrer Mitarbeiter_innen haben die Träger das Personal abgezogen bzw. werden diese aus Gründen des Infektionsschutzes nicht mehr in die Anstalten gelassen. Vereinzelt wird ehrenamtlichen Betreuer_innen genehmigt, die Betreuung von Gefangenen weiterzuführen.

Die Durchführung von Behandlungsangeboten ist zu Pandemiezeiten sekundär. Das Angebot resozialisierender Maßnahmen kann nicht im gewohnten Umfang aufrechterhalten werden. Die bei Wahrung des Infektionsschutzes bestehenden Möglichkeiten sind zu begrenzt, die Köpfe dafür weder bei Gefangenen noch Bediensteten frei. Es geht in den Anstalten darum, die Situation unter Kontrolle zu haben. Die Gefangenen nehmen die Angebote zur Krisenintervention bislang gerne an. Vielleicht ist es ein Nebeneffekt einer gemeinsam bewältigten Krise, dass sich die Distanz zwischen Bediensteten und Inhaftierten verringert.

\section{Übergangsmanagement: Wie? Mit wem?}

Der Sozialdienst versteht sich als Teil der Straffälligenhilfe $^{9}$. Zu diesem Verständnis gehört es, Inhaftierten die Angebote der freien und öffentlichen-rechtlichen Träger zu erschließen und im Rahmen des Übergangsmanagements strukturbezogene Netzwerke zur sozialen und be- 
ruflichen Integration von Inhaftierten/Haftentlassenen aufzubauen ${ }^{10}$. Das Übergangsmanagement, das als gemeinsame Aufgabe aller intern und extern Beteiligten zu verstehen ist, bietet den Rahmen für die einzelfallbezogene Entlassungsvorbereitung.

Die Integration Haftentlassener ist angewiesen auf externe Netzwerke, wie z. B. mit der Suchtkranken-, Wohnungslosen- und Schuldnerhilfe, mit Arbeitsmarktakteuren sowie mit öffentlich-rechtlichen Trägern. Dies trifft insbesondere auf Flächenstaaten wie NordrheinWestfalen zu, in denen Gefangene häufig heimatfern inhaftiert sind. Es gilt umso mehr, als dass aufgrund der Haftunterbrechung durch die Sozialdienste binnen kürzester Zeit Hunderte von Fällen zu prüfen und Gefangene insbesondere in Hinblick auf die Sicherung der Unterkunft, der medizinischen Versorgung und des Lebensunterhalts auf eine zeitnahe Entlassung vorzubereiten waren.

Mit Beginn der Corona-Krise brachen die Netzwerke zunächst sukzessive und später auf breiter Ebene weg. In dieser Phase war jede_r Sozialarbeiter_in gefordert, aufwendig in jedem Einzelfall Lösungen zu finden. Lösungen, die gestern noch möglich waren, heute aber verworfen sind und morgen ganz anders aussehen konnten. Es gab keine Linie, keine Verlässlichkeit, keine Orientierung. Jede Behörde, jeder Träger verfuhr anders. Viele Mitarbeiter_innen fühlten sich allein gelassen. Um einen gewissen Überblick zu erhalten, begannen dann einige Sozialdienste systematisch Abfragen bei den externen Kooperationspartnern zu starten, regelmäßig aktualisierte Übersichten zu den sich stetig ändernden Leistungen zu erstellen, die dann landesweit zur Verfügung gestellt wurden. Den zu Entlassenen wurden selbstgestrickte Anträge auf Sozialleistungen mitgegeben und tagesaktuelle Informationsblätter ausgehändigt.

\section{Zentrale Bereiche des Übergangsmanagements brechen weg}

Obwohl die Bundesagentur für Arbeit Mitte März das Antragverfahren vereinfacht und flexibilisiert hatte, stellte sich als größtes Problem die Sicherstellung des Lebensunterhalts heraus. Jobcenter und Arbeitsagenturen blieben für den Publikumsverkehr geschlossen. Telefonleitungen waren aufgrund der Nachfragen zum Kurzarbeitergeld überlastet. Die Möglichkeit, Leistungen online zu beantragen, haben die meisten Haftentlassenen nicht. Telefonisch wurde den Mitarbeiter_innen der Sozialdienste zugesichert, dass alle Anträge - auch postalisch gestellte - zeitnah bearbeitet würden. Die Auskünfte darüber, wie der Leistungsanspruch im Fall einer Haftunterbrechung sich gestaltet, variierten zudem von Behörde zu Behörde. Die eine sah keinen Leistungsan- spruch auf ALG II gegeben, die andere erkannte ihn an, da Vermittlungsmöglichkeiten aufgrund der Nachfrage nach Saisonarbeitern bestehen. Als das Gros der Gefangenen entlassen war, erreichte die Anstalten die Information, dass die Bundesanstalt für Arbeit keinen Leistungsanspruch bei Haftunterbrechung begründet sah, da die Entlassenen den Strafrest noch verbüßen müssen und deshalb dem Arbeitsmarkt nicht zur Verfügung stehen würden. Ein Anspruch nach dem SGB XII solle geltend gemacht werden.

Auf einem leergefegten Wohnungsmarkt für zur Entlassung anstehende Gefangene eine Unterkunft zu finden, das ist schon zu „normalen“ Zeiten fast unmöglich - und erst Recht in Zeiten von Corona. Vereinzelt verzichten Gefangene auf Entlassungen, um ihre betagten Eltern nicht zu gefährden. Anfragen bei Wohneinrichtungen ergaben eine sehr unterschiedliche Praxis. Der eine Teil der Einrichtungen nimmt unvermindert auf, obwohl das notwendige vorherige persönliche Kennenlernen im Rahmen von vollzugsöffnenden Maßnahmen derzeit nicht möglich ist. Die Aufnahmemodalitäten werden telefonisch abgesprochen. Der andere Teil hat einen Aufnahmestopp ausgesprochen, um Bewohner_innen und Personal vor Infektionen zu schützen. Gelegentlich besteht die Option für eine Einzelfallentscheidung. Vereinzelt konnten zuvor vereinbarte Aufnahmetermine nicht gehalten werden. Bei den kommunalen Notunterkünften ergibt sich ein ähnliches Bild: manche sind voll belegt, einige weisen noch zu, andere haben die Platzkapazitäten aufgrund der in Mehrbettzimmern bestehenden Ansteckungsgefahren verringert. Die Beratungsstellen für Haftentlassene und ihre Familien begleiten keine Klienten mehr; sie verrichten z.T. Notdienste und bieten stundenweise telefonische Beratungen an. In der aktuellen Notsituation stellen viele Träger die Anschrift ihrer Einrichtung als postalische Adresse zur Verfügung, um den Hilfesuchenden die Möglichkeit zu schaffen, postalisch für Behörden, z. B. für die Beantragung von Arbeitslosengeld, erreichbar zu sein.

Viele Gefangene sind nach der Entlassung auf medizinische Hilfen angewiesen. Dies trifft besonders auf die hohe Zahl inhaftierter Drogenabhängiger zu, die unter Suchtfolgekrankheiten leiden, deren Immunsystem angegriffen ist und die deshalb zu den Risikogruppen zählen. Nahtlos - also unmittelbar am Entlassungstag eine Krankenversicherung sicherzustellen, das ist von jeher praktisch unmöglich. Erst muss der Antrag auf ALG gestellt und bewilligt sein, damit erfolgt die Anmeldung bei der Krankenkasse. Vor der Antragstellung muss der Entlassene sich ausweisen können und über eine Entlassungsadresse bzw. Anmeldung verfügen. Insofern ist es 
wie immer: die unmittelbare medizinische Versorgung kranker Haftentlassener ist nicht sichergestellt.

$\mathrm{Da}$ die Mitarbeiter_innen externer Träger nicht mehr in den Anstalten tätig sind, sind besonders die Angebote im Rahmen der Suchtberatung eingeschränkt. Dies betrifft die Therapievermittlung und das Übergangsmanagement zu Einrichtungen des regionalen Suchthilfesystems. Die Substitution von Drogenabhängigen nach der Entlassung ist durch den Goodwill behandelnder Ärzt_innen, die in Vorleistung gehen, häufig gesichert. Therapieeinrichtungen folgen zumeist der Empfehlung der Deutschen Rentenversicherung, keine Neuaufnahmen mehr durchzuführen. Auch wenn noch einzelne Aufnahmen mit der Vorgabe erfolgen, dass der medizinische Dienst der Anstalten Infektionsfreiheit bescheinigt, platzen Aufnahmetermine mit der Folge einer weiteren Inhaftierung. Corona hebelt den $\$ 35$ BtMG (Therapie statt Strafe) aus.

Eintrittskarte für alle Hilfen im Übergangsmanagement ist ein gültiger Ausweis. Er ist Grundlage für die Anmeldung und für die Beantragung von Sozialleistungen. Da die Stadtverwaltungen den Öffentlichkeitsverkehr weitgehend eingestellt haben und Gefangene nicht mehr zu den Passstellen ausgeführt werden, werden keine Personalausweise mehr ausgestellt. Gefangene werden darauf verwiesen, nach der Entlassung bei den Meldeämtern einen vorläufigen Ausweis mit einer Gültigkeitsdauer von drei Monaten zu beantragen.

\section{Die Ärmsten und Hilfebedürftigsten trifft es am härtesten}

Die Corona-Pandemie trifft Inhaftierte und Haftentlassene in besonderem Maße. Inhaftierte sind in ihren Handlungsmöglichkeiten stark eingeschränkt, können nicht einfach mal so anrufen, vorsprechen oder per eMail Nachfragen stellen. Viele bedürfen der Vorsprache, Begleitung und persönlichen Unterstützung. Genau diese sozialen Dienstleistungen sind weggebrochen. Die Corona-Krise hat massive Auswirkungen auf die Entlassenen, die auf intensive Unterstützung angewiesen sind: die Hilflosen, Überforderten, Unselbständigen, die, die keine Kontakte haben, die Kranken und die Armen.

\section{Nach Corona: was dann?}

Nach den Erfahrungen der vollzuglichen Sozialdienste waren alle Mitarbeiter_innen bei Behörden und Trägern hilfsbereit und gewillt, konstruktive und auch kreative Lösungen zu finden. Die widersprüchlichen Informationen, die von der Zeit überholten Auskünfte waren der Unsicherheit und der Hilflosigkeit in einer bisher nicht dagewesenen Situation geschuldet. Alle Beteiligten - intern wie extern - haben nach bestem Wissen und Gewis- sen ihre Entscheidungen getroffen. Kritik an Einzelentscheidungen ist aufgrund der Ausnahmesituation nicht angebracht. Dennoch muss die Frage erlaubt sein, was wir aus Corona für die Zeit nach Corona und für weitere Krisen lernen können.

Corona zeigt uns Grenzen auf, Grenzen im Einzelfall, aber auch Grenzen im System. Es darf nicht sein, dass der Hilfe bedürftige Gefangene in ungeklärte Situationen entlassen werden und keine Unterstützung erfahren. Er darf aber auch nicht sein, dass einzelne Mitarbeiter_ innen - gleichgültig, ob im Vollzug, in der Bewährungshilfe oder bei freien Trägern tätig - aufwendig und frustrierend in jedem Einzelfall neu klären muss, wie jemand einen Personalausweise bekommt, ob und wo jemand einen Leistungsanspruch hat oder ob und wann welche Krankenversicherung jemanden aufnimmt. Diese beispielhafte Aufzählung zeigt die gravierenden Mängel im System, die die Corona-Pandemie deutlich fokussiert hat. Alle Bereiche treffen für sich isoliert Entscheidungen. Die Integration Haftentlassener ist aber eine gesamtgesellschaftliche Aufgabe ${ }^{11}$. Diese wird auf dem Rücken der Betroffenen und Mitarbeiter_innen ausgetragen, statt sich mit dem ernsthaften Willen an einen Tisch zu setzen und Lösungen zu wollen, indem formales Zuständigkeitsdenken überwunden wird und rechtliche Bestimmungen modifiziert und übergreifende Kooperationsvereinbarungen geschlossen werden. In der Krise liegt die Chance. Wenn Corona dazu führen sollte, endlich die allseits bekannten übergreifenden Probleme in gesamtgesellschaftlicher Verantwortung zu lösen, wäre der Krise etwas Positives abzugewinnen.

1. Z. B.: Ministerium der Justiz des Landes Nordrhein-Westfalen. Infektionsgefährdung von Gefangenen und Bediensteten durch das neuartige Coronavirus; Erlass vom 09.03.2020.

2. Ministerium der Justiz des Landes Nordrhein-Westfalen. Vollstreckungsaufschub für Ersatzfreiheitsstrafe gemäß \$ 455 a StPO aus Anlass der Corona-Pandemie; Erlass vom 17.03.2020.

3. Ministerium der Justiz des Landes Nordrhein-Westfalen. Vollstreckungsaufschub bei kurzen Freiheitsstrafen sowie Unterbrechung der Vollstreckung von Ersatzfreiheitsstrafen und kurzen Freiheitsstrafen aus Gründen der Vollzugsorganisation gem. \$455a StPO aus Anlass der Corona-Pandemie; Erlass vom 20.03.2020.

4. Ministerium der Justiz des Landes Nordrhein-Westfalen. Infektionsgefährdung von Gefangenen und Bediensteten durch das neuartige Coronavirus; Erlass vom 16.03.2020.

5. Schlebusch, Stephan (2017). Überlegungen zum Selbstverständnis Sozialer Arbeit im Justizvollzug. In: 50 Jahre LAG; S. 20-36, S. 31; http://www.lag-sozialdienst-nrw.de/pdf/50\%20Jahre\%20LAG.pdf; zugegriffen: 19. April 2020.

6. JM-NRW, Justizminister des Landes Nordrhein-Westfalen (2017). Richtlinien für die Fachdienste bei den Justizvollzugseinrich- 
tungen des Landes Nordrhein-Westfalen-AV d. JM vom 18. Dezember 2015 (2400 - IV. 54) - JMBl. NRW S. 3 in der Fassung vom 06. Juli 2017 - JMBl. NRW S. 198.

7. Nr. 2.6.4.1 der Richtlinien für die Fachdienste bei den Justizvollzugseinrichtungen des Landes Nordrhein-Westfalen; JM-NRW, Justizminister des Landes Nordrhein-Westfalen (2017). Richtlinien für die Fachdienste bei den Justizvollzugseinrichtungen des Landes Nordrhein-Westfalen-AV d. JM vom 18. Dezember 2015 (2400IV. 54) - JMBl. NRW S. 3 in der Fassung vom 06. Juli 2017 - JMBl. NRW S. 198.

8. Vgl. die Beschreibung der Aufgaben des vollzuglichen Sozialdienstes durch: Empt, Yvonne u. a. (2020). Erwachsenenstrafvollzug. In: Deimel, Daniel und Köbler, Torsten. Delinquenz und Soziale Arbeit (S. 241-254). Lengerich: Pabst.

9. Schlebusch, Stephan (2017), a. a. O., S. 31.

10. Zur Begriffsdefinition s.: Wirth, Wolfgang (2019). Entlassung vorbereiten und Eingliederung gestalten. In: Forum Strafvollzug, 68. Jahrgang, Heft 4/19, S. 260.

11. Vgl. Baum, Rudolf (2018). Übergangsmanagement als gesamtgesellschaftliche Aufgabe. In: Reichenbach, Marie-Therese und Bruns, Sabine: Resozialisierung neu denken (S. 125-133). Freiburg: Lambertus.

Hier steht eine Anzeige.

Springer 